\title{
Experimental Investigation of Ion Mobility Measurements in Oxygen under Different Gas Pressures
}

\author{
Yun-Peng Liu* and Shi-long Huang ${ }^{\dagger}$
}

\begin{abstract}
In this paper, measurements of ion mobility were performed in oxygen at gas pressures of 44.52 - $101.19 \mathrm{kPa}$ using the drift tube method. Over this pressure range, mobility values were within the limits of 1.796 to $3.821 \mathrm{~cm}^{2} \cdot \mathrm{V}^{-1} \cdot \mathrm{s}^{-1}$ were determined and ion mobility shown to decrease nonlinearly with increasing gas pressure towards a certain level of saturation. Ion mobility measured in air was lower than that measured in oxygen at the same gas pressure. Finally, a parameter correction method for calibrating the relationship between the ion mobility and gas pressure in oxygen was proposed.
\end{abstract}

Keywords: Gas pressure, Correction method, Negative corona discharge, Ion mobility, Oxygen

\section{Introduction}

The solution to certain problems of plasma physics and electronics is connected to the determination of mobility and the nature of charged particles in gas media [1]. Whilst the existing value of the ion mobility is not dependent on gas pressure [2-4], oxygen is the main reaction gas in negative corona discharge. Measurements of ion mobility in oxygen are of significant importance and much remains to be determined concerning the specific mechanism of corona discharge.

According to the Mason-Schamp ion mobility Eq. (1) [5] :

$$
\mu=\frac{3}{16} \frac{\mathrm{Ze}}{\mathrm{N}}\left(\frac{2 \pi}{m M /(\mathrm{m}+M) \cdot k T}\right)^{1 / 2} \frac{(1+\alpha)}{\Omega_{0}}
$$

In (1), $Z$ is ion charge number, $e$ is electronic charge, $N$ is the molecular number of drift gas $\left(\mathrm{n} / \mathrm{cm}^{3}\right), m$ is the ion mass, $M$ is the molecular mass of drift gas, $k$ is disposal of Boltzmann's constant, $T$ is kelvin temperature, $a$ is a correction term, $\Omega_{0}$ is collision scattering angle and average energy correction term. It can be seen that ion mobility is directly related to the gas pressure (drift gas density $N$ ) and absolute temperature $T$.

Methods for measuring the mobility spectra of atmospheric ions have been put forward by a number of investigators and include the Gerdien condenser method [6, $7]$, the drift tube method $[8-16,25,26]$, the parallel plate electrodes method $[17,18]$, the pulse method [20], and the voltage-current curve method [21, 22]. The test of the voltage-current curve method is simplistic and has a

$\dagger \quad$ Corresponding Author: Department of Electrical Engineering, North China Electric Power University, China. (wzhp_hsh19999@163.com)

* Department of Electrical Engineering, North China Electric Power University, China. (liuyunpeng@ncepu.edu.cn)

Received: August 22, 2016; Accepted: November 18, 2016 similar measurement result in practical engineering but lacks theoretical basis as the electric field distribution after inception of the corona and the development of corona in severe non-uniform electric field is unclear so far. The Pulse method has a much stricter requirement of the test equipment and conditions compared to the drift tube method. The Drift tube method has gradually developed into the ion mobility spectrum analysis method; the underlying theory of which is relatively mature, therefore the drift tube method was adopted in this paper.

Tuong [23] investigated the influence of oxygen vacancy's concentration on the ion mobility. Kosarim [24] developed a method to distinguish the atomic oxygen ions in the ground and in metastable states according to their different mobility. Hill [25] used a pulsed corona discharge structure as the ion source to obtain atmospheric chemical ionisation mass spectra and selected-mass mobility spectra. Ebrahimi [26] proposed a method based on negative corona discharge ionization ion mobility spectrometry (NCD-IMS) to specify the sample extracted via dispersive liquid-liquid microextraction (DLLME). Cumeras [27] reviewed the structure and function of all the current IMS instrumentations. Sabo [28, 29] studied the ion mobility spectrometry in oxygen to monitor high-purity oxygen mixed with foreign gas, and measured corona discharge ion mobility spectra of high purity $\mathrm{O}_{2}$ and $\mathrm{O}_{2} / \mathrm{N}_{2}$ mixtures in the atmospheric pressure using the drift tube method with the primary ions $\mathrm{O}^{-}, \mathrm{O}^{2-}$ and $\mathrm{O}^{3-}$ detected in the negative corona in pure $\mathrm{O}_{2}$. Urquijo [30] measured the mobility of a single negative ion species in oxygen at high gas pressures of 20.7 and $68.9 \mathrm{kPa}$ using the pulse method and obtained a mean mobility value of $2.16 \pm 0.08$. However, the experimental data reported is relatively old and was carried out in low pressure drift tubes and at higher values of the reduced electric fields $\mathrm{E} / \mathrm{N}$. To date, there has been no systematic measurement of ion mobility in oxygen under different gas pressure. 
In this paper, the experimental platform measuring ion mobility was built based on corona discharge model using the drift tube method. Ion mobility at different gas pressures $(44.52-101.19 \mathrm{kPa})$ in oxygen was measured and provides a reference for the analysis on the corona discharge characteristics and has significance for the study of negative corona discharge and plasma physics.

\section{Ion Mobility Measurement Tests}

\subsection{The construction of the experimental platform}

A schematic drawing of the experimental setup is presented in Fig. 1, and a diagram of the experimental platform shown in Fig. 2. The ion mobility measurement device based on corona discharge mainly consisted of three parts: a corona discharge ionization zone (A), an ion migration area (B) and a charge collection area (C). In the corona discharge ionization zone, the needle-ring corona

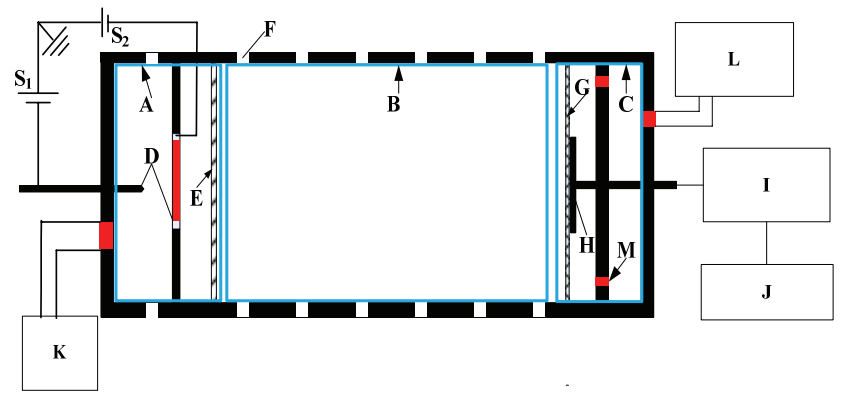

Fig. 1. A schematic drawing of the experimental setup.

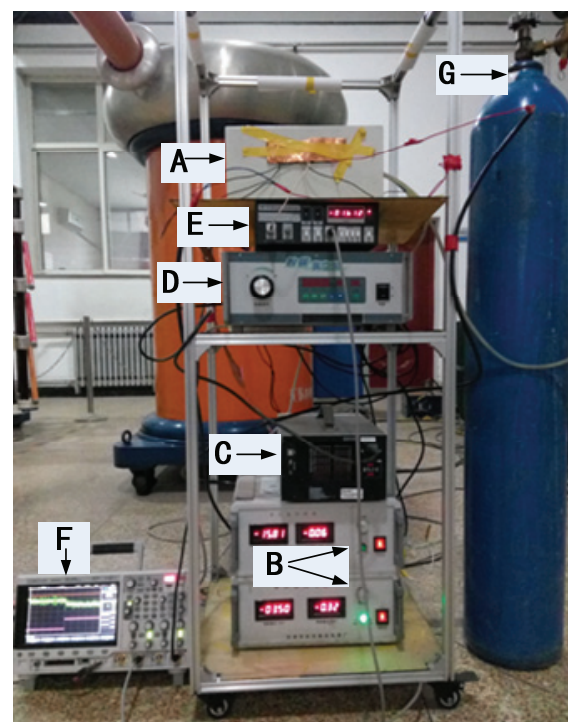

Fig. 2. Measuring platform. The platform consisted of shielding box with drift tube (A), HVDC power supply (B), the power supply of control circuit (C), air sucking pump (D), the micro current amplifier $(\mathrm{E})$, the oscilloscope (F), and the oxygen container (G). discharge structure (D) is used as an ion source. The needle ring has a fixed spacing of $4.3 \mathrm{~mm}$ and the voltage applied to the needle and ring was $-7.5 \mathrm{kV}$ and $-1.35 \mathrm{kV}$ respectively, which was generated by the HDVC power supply (S1 and S2). The Bradbury-Nielson metal wire ion gate (E) was used [31, 32] and the execution of the ion gate was improved from the off-on-off to off-on to allow a higher ion current amplitude. In the migration area, the outer wall of the drift tube was arranged by several stainless steel rings $(\mathrm{F})$ and a series resistance of $1 \mathrm{M} \Omega$ is set between the rings to ensure that the electric field in drift tube is uniform as far as possible. The length of drift tube was $10 \mathrm{~cm}$ so the electric field in drift tube is $135 \mathrm{~V} / \mathrm{cm}$. In the charge collection area, an aperture grid $(G)$ was attached to the last ring by $2200 \mathrm{pf}$ capacitor in front of a Faraday plate detector $(\mathrm{H})$ center-to-center to filter the high-frequency interference current caused by the ion which are close to but do not arrive at the faraday plate which is used to gather ion current signal coming from drift area. Due to the ion current signal, the level is only $\mathrm{nA}$ and a micro current amplifier (I) was used to convert current into a voltage signal obtained by digital memory oscillograph $(\mathrm{J})$. The drift tube cavity was made of PTFE material and the joint parts are sealed using Kafuter blue glue. The gas pressure in the drift tube was controlled by an air sucking pump (K) and an air sending pump (L) cooperatively through the air hole (M).

The altitude of the transmission line in China is $14 \mathrm{~m}$ in a flat area and $6000 \mathrm{~m}$ in Tibet. According to the relationship between gas pressure and altitude in Eq. (2), the gas pressure points measured are as follows in Table 1 correspondingly.

$$
p=p_{0} \cdot\left(1-\frac{A}{k}\right)
$$

Table 1. Simulated Altitude and gas pressure

\begin{tabular}{c|c|c|c}
\hline$A / \mathrm{km}$ & $p / \mathrm{kPa}$ & $A / \mathrm{km}$ & $\mathrm{p} / \mathrm{kPa}$ \\
\hline 0.014 & 101.19 & 3.500 & 68.18 \\
\hline 0.500 & 96.59 & 4.000 & 63.45 \\
\hline 1.500 & 87.12 & 5.000 & 53.98 \\
\hline 2.000 & 82.39 & 5.500 & 49.25 \\
\hline 2.500 & 77.65 & 6.000 & 44.52 \\
\hline 3.000 & 72.92 & $/$ & $/$ \\
\hline
\end{tabular}

Where $p$ is the pressure at an altitude of $A(\mathrm{~km}), p_{0}$ is the pressure at sea level $(101.325 \mathrm{kPa})$ and $k$ is an empirical constant (10.7) [33].

\subsection{Ion gate and control circuit}

\subsubsection{The bradbury-nielson metal wire ion gate}

The diagram of the Bradbury-Nielson metal wire ion gate used is shown in Fig. 3. $U_{a}$ and $U_{b}$ are the potentials of the ion gate terminal ' $a$ ' and ' $b$ ' respectively. The working 


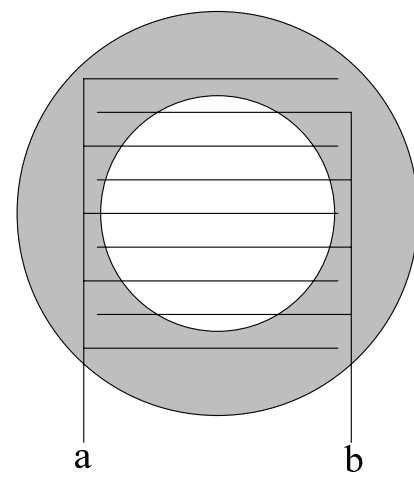

Fig. 3. Diagram of the Bradbury-Nielson metal wire ion gate, ' $a$ ' and 'b' are two terminals of the ion gate

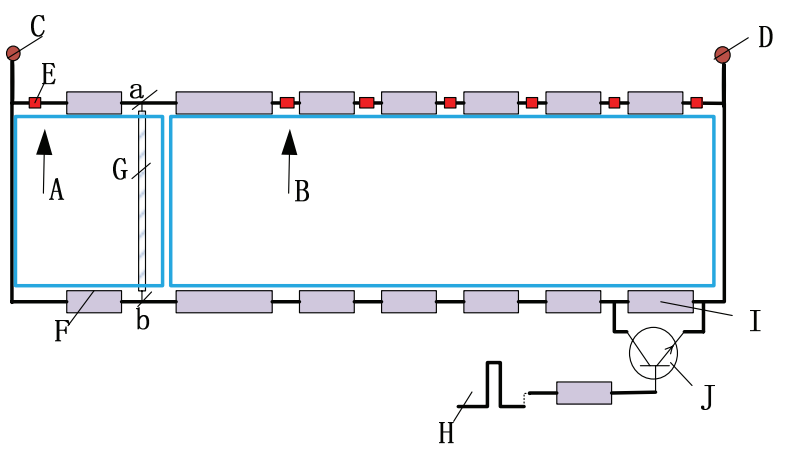

Fig. 4. Diagram of the Ion gate control circuit

principle of ion gate is that when $\mathrm{U}_{\mathrm{a}} \neq \mathrm{U}_{\mathrm{b}}$, adjacent wires will have a potential difference which produces a perpendicular electric field to the ion migration direction, and the ion will collide with ion gate wire and be neutralized. Therefore, the ion current at this time will not be measured and the ion gate is closed. When the ion gate is fully open, the potential difference between the adjacent wires disappears and the ion will enter migration area and arrive at Faraday plate.

\subsubsection{Control circuit of the ion gate}

In this paper, the series resistance value changed to control the opening and closing of the ion gate [19], is shown in Fig. 4. The main function area of the ion gate control circuit includes a corona discharge ionization zone (A) and the ion migration area (B). $-1.35 \mathrm{kV}$ is set between the high voltage terminal (C) and the grounding terminal (D) to generate an electric field along the ion immigration direction. The outer wall of the drift tube was arranged by several stainless steel rings $(\mathrm{E})$, Resistance $(1 \mathrm{M} \Omega)$ in series (F) was added and connected outside the drift tube between the rings to ensure the electric field in the drift tube was uniform as far as possible. The ion gate execution was controlled by the potential difference between terminals ' $a$ ' and ' $b$ ' which can be achieved by adjusting the resistance value connected to the terminal ' $b$ ' of the ion gate. When the pulse $(\mathrm{H})$ was at a low level, the voltage values

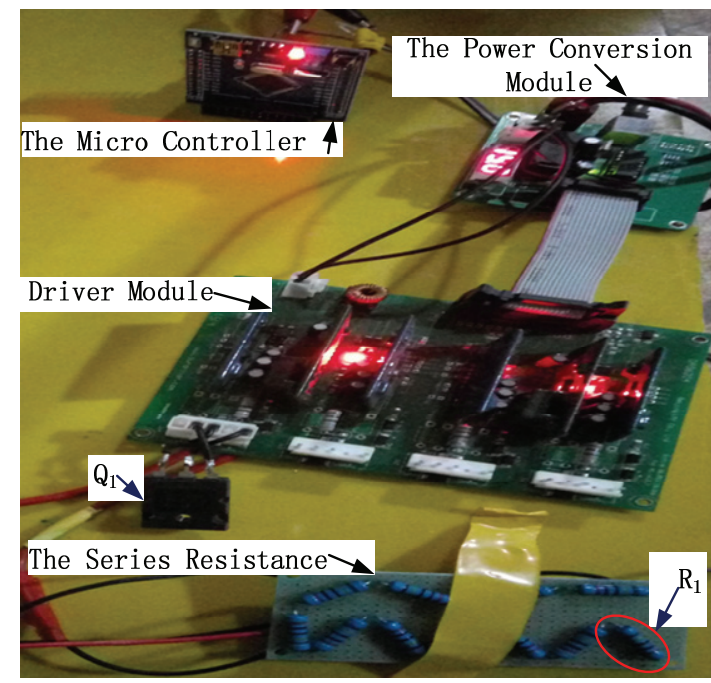

Fig. 5. The Ion gate control circuit. The power conversion module was used to provide the power supply for the micro controller STM32F107 and the driver module. The pulse is generated by the microcontroller which is amplified and optical-coupling isolated by the driver module to control the transistor $\mathrm{Q}_{1}$ and $\mathrm{R}_{1}$ ' short

between terminals ' $a$ ' and ' $b$ ' are equal and there was no component perpendicular to the immigration electric field and ions could go through into the drift area when the ion gate was open. However, when the pulse was at a high level, the resistor $\mathrm{R}_{1}(\mathrm{I})$ was short and there was a potential difference between ' $\mathrm{a}$ ' and ' $\mathrm{b}$ ' along the vertical direction to prevent ions from migrating into the area, so the ion gate was close. The controlling of $\mathrm{R}_{1}$ 's short is operated by a transistor $\mathrm{Q}_{1}(\mathrm{~J})$, and the pulse was generated by the microcontroller STM32F107. A schematic of the control circuit is presented in Fig. 5.

\subsection{Corona discharge ion analysis in oxygen}

The primary electron is ionized from the high intensity electric field of the needle electrode in the corona discharge. A positive ion and a secondary electron are generated during the collision between the primary electron and oxygen molecule. The primary and secondary electron repeat the above process until their energy is lower than the energy needed to ionize oxygen molecules. In the process, the positive ions produced by the impact ionization move slowly and enter the needle. Finally, in the region near the needle, the electric field strength significantly weakens and the electrons migrate at a lower velocity and attach on to oxygen molecules forming negative ions. Meanwhile, the negative corona discharge in $\mathrm{O}_{2}$ is a source of neutral molecules and radicals, of which the most important is $\mathrm{O}_{3}^{-}$. The generation of $\mathrm{O}_{3}{ }^{-}$is shown in the ion equations below (3)-(6). In the process of generating a negative polarity corona in oxygen, the final products are $\left[\left(\mathrm{H}_{2} \mathrm{O}\right)_{\mathrm{n}} \mathrm{O}\right]^{-}$, 
$\left[\left(\mathrm{H}_{2} \mathrm{O}\right)_{\mathrm{n}} \mathrm{O}_{2}\right]^{-}$and $\left[\left(\mathrm{H}_{2} \mathrm{O}\right)_{\mathrm{n}} \mathrm{O}_{3}\right]^{-}$. Due to different moisture content of the gas, the value of $n$ is $1,2,3$.

$$
\begin{gathered}
2 \mathrm{O}_{2}+e^{-} \rightarrow \mathrm{O}_{2}^{-}+\mathrm{O}_{2} \\
\mathrm{e}^{-}+\mathrm{O}_{2} \rightarrow O^{-}+O \\
O^{-}+O_{3} \rightarrow O_{3}^{-}+O \\
O_{2}^{-}+O_{3} \rightarrow O_{3}^{-}+O_{2}
\end{gathered}
$$

\section{Analysis of Measured Results}

In this experiment, the oxygen purity was $99.99 \%$. Before the measurements, the air sucking pump and air sending pump are used in cooperation to inject oxygen into the drift tube for 10 minutes to remove the foreign gas and guarantee the purity of the oxygen in the drift tube

As the ion gate opens as a starting point in time, the drift time $t$ stands for the time when the ion current flow into the Faraday plate, as is shown in Fig. 6 (gas pressure of 101.19 $\mathrm{kPa}$ in oxygen), $t=t_{1}+t_{2}, t_{1}$ is affected by the ion collision frequency with other particles in the process of transport, which is influenced by gas pressure; $t_{2}$ is affected by the hydrated complex degree, which is influenced by temperature and humidity. $L$ and $E$ are the length and electric field of the drift tube, so the ion mobility $k$ is:

$$
k=v / E=L /\left(t^{*} E\right)
$$

In the experiments, the measuring points of the gas pressure started from the highest pressure to prevent the

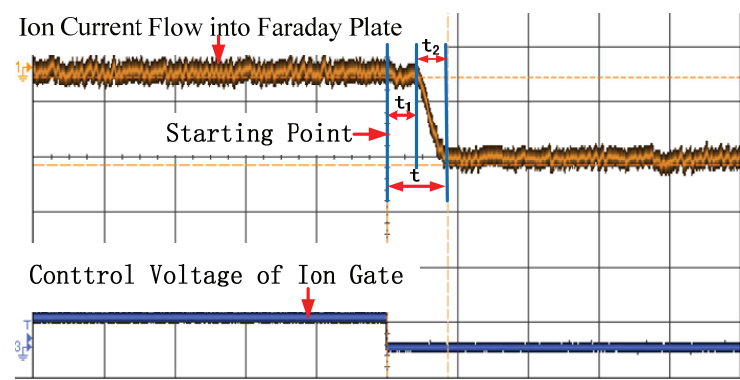

Fig. 6. Measurement of drift time $t$

Table 2. The difference of ion mobility in air and oxygen

\begin{tabular}{c|c|c|c}
\hline $\boldsymbol{p} / \mathrm{kPa}$ & $\boldsymbol{k}$ in air $/ \mathrm{cm}^{2} \mathrm{~V}^{-1} \mathrm{~s}^{-1}$ & $\boldsymbol{K}$ in oxygen $/ \mathrm{cm}^{2} \mathrm{~V}^{-1} \mathrm{~s}^{-1}$ & The $\delta$ value \\
\hline 44.52 & 3.398 & 3.821 & 0.423 \\
\hline 49.25 & 2.955 & 3.346 & 0.391 \\
\hline 53.98 & 2.665 & 2.910 & 0.245 \\
\hline 63.45 & 2.228 & 2.396 & 0.168 \\
\hline 68.18 & 2.158 & 2.278 & 0.12 \\
\hline 72.92 & 2.075 & 2.168 & 0.093 \\
\hline 77.65 & 1.942 & 2.061 & 0.119 \\
\hline 82.39 & 1.788 & 1.980 & 0.192 \\
\hline 87.12 & 1.754 & 1.904 & 0.15 \\
\hline 96.59 & 1.608 & 1.817 & 0.209 \\
\hline 101.19 & 1.562 & 1.796 & 0.234 \\
\hline
\end{tabular}

foreign gases spreading into the drift tube. The temperature and relative humidity of the experimental platform were

(a)

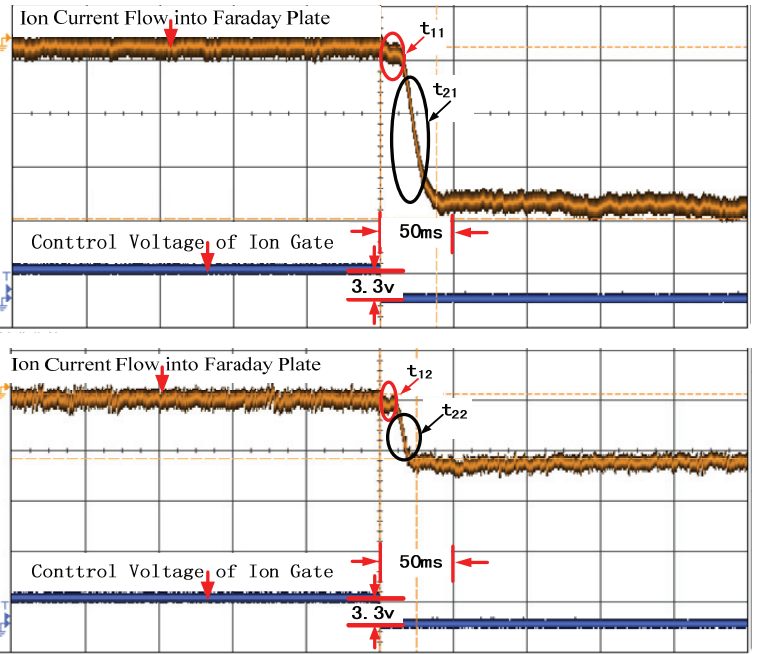

(c)

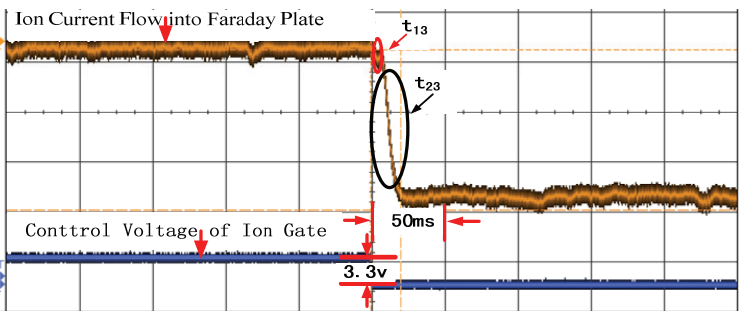

Fig. 7. Ion mobility spectra under gas pressures of (a) 91.86 ; (b) 63.45 ; (c) $44.52 \mathrm{kPa}$ in oxygen.

(a)
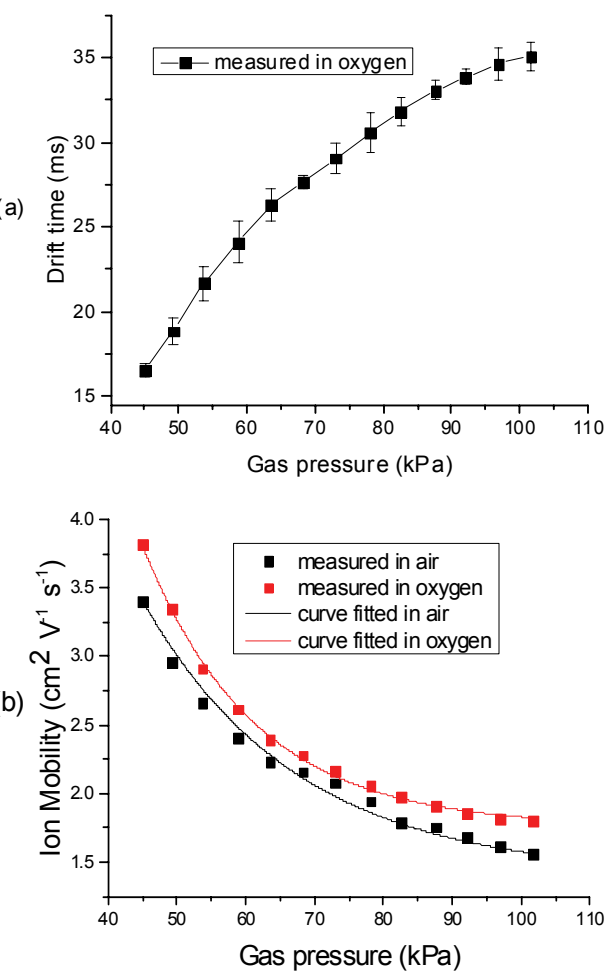

Fig. 8. (a) Ion migration under different gas pressures in oxygen; (b) Ion mobility under different gas pressures in air and oxygen 
$15-17{ }^{\circ} \mathrm{C}$ and $30-40 \%$ respectively. A gas pressure of 44.52-101.19 kPa were preliminary measured and the drift time under different pressures was obtained through more than ten repeated measurements, as shown in Fig. 7 and Fig. 8. From Fig.7, it can be seen that $t_{11}>t_{12}>t_{13}$ and $t_{21} \approx$ $\mathrm{t}_{22} \approx \mathrm{t}_{23}$ as only the mean free path of the ion increases with the decrease in gas pressure, which decreases the drift time.

It can be seen that ion drift time in oxygen increases non-linearly with the increase of gas pressure but has a trend towards saturation as shown in Fig. 8(a). The ion mobility is negatively correlated with ion drift time, thus the ion mobility in oxygen decreases non-linearly with the increase in gas pressure and has a trend towards saturation.

The ion mobility in air has previously been measured in [15]. The ion mobility in air is lower than that of measured in oxygen, and the difference of ion mobility in air and oxygen is shown in Table 2. In addition, the mobility changing rate in oxygen is smoother than that in air. One possible reason giving rise to the result described above is that the gas composition in air is more complicated than that in oxygen. The reaction product [25, 28, 29], does not only contain $\left[\left(\mathrm{H}_{2} \mathrm{O}\right)_{n} \mathrm{O}\right]-,\left[\left(\mathrm{H}_{2} \mathrm{O}\right)_{n} \mathrm{O}_{2}\right]-,\left[\left(\mathrm{H}_{2} \mathrm{O}\right)_{n} \mathrm{O}_{3}\right]-$, but also can generate $\left[\left(\mathrm{H}_{2} \mathrm{O}\right)_{n} \mathrm{CO}_{3}\right]-,\left[\left(\mathrm{H}_{2} \mathrm{O}\right)_{\mathrm{n}} \mathrm{HCO}_{3}\right]-$, $\left[\left(\mathrm{H}_{2} \mathrm{O}\right)_{\mathrm{n}} \mathrm{NO}_{3}\right]-$, therefore under the same gas pressure, the volume, proton mass and $\mathrm{m} / \mathrm{z}$ of negative ions produced by a corona discharge in air is slightly bigger than that in oxygen.

The ion mobility measured in oxygen is non-linearly fitted, and a good effect is obtained when using exponential fitting. The fitting results are shown in Fig. 8. (b) and the fitting function shown in Eq. (8), and the corresponding fitting correlation coefficient calculated as 0.9722 .

$$
K=1.7596+32.9361 \mathrm{e}^{-0.0617 P}
$$

In Eq. (8), $K$ is ion mobility, $P$ is gas pressure.

\section{Conclusions}

1) The ion mobility in oxygen was obtained under gas pressures of $44.52-101.19 \mathrm{kPa}$, and the negative ion mobility measured was in the range of $1.796-3.821$ $\mathrm{cm}^{2} \cdot \mathrm{V}^{-1} \cdot \mathrm{s}^{-1}$.

2) The ion mobility in oxygen decreases non-linearly with increasing gas pressure and has a certain trend towards saturation.

3) The ion mobility in air is lower than that of measured in oxygen, and the $\delta$ value is within the limits of $0.093-0.423$.

4) A parameter correction method for calibrating the relationship between ion mobility and gas pressure in oxygen has been proposed.

\section{Acknowledgements}

This project was supported by the National Natural Science Foundation of China(51577069).

\section{References}

[1] R. I. Garber, V. D. Partsyrnyi, and I. I. Soloshenko, "Use of an open proportional counter to determine oxygen-ion mobility in air," Russian Physics Journal, vol. 14, no. 11, pp. 1560-1561, 1971.

[2] M. Sarma, W. Janischewskyj, "Analysis of corona losses on DC transmission lines part II-bipolar lines," IEEE Transactions on Power Apparatus and Systems, vol. 88, no. 10, pp. 1476-1491, 1969.

[3] T. Takuma, T. Ikeda, and T. Kawamoto, "Calculation of ion flow fields of HVDC transmission lines by the finite element method," IEEE Transactions on Power Apparatus and Systems, vol. 100, no. 12, pp. $4802-$ 4810, 1981.

[4] W. Li, B. Zhang, and J. L. He, "Ion flow field calculation of multi-circuit DC transmission lines," in International Conference on High Voltage Engineering and Application, 2008, pp. 16-19.

[5] E. A. Mason, and E. W. McDaniel, Transport Properties of Ions in Gases. New York: Wiley, 1988.

[6] E. C. Whipple, "An improved technique for obtaining atmospheric ion mobility distributions," Journal of Geophysical Research, vol. 65, no. 11, pp. 367-3684, 1960.

[7] K. Suzuki, M. Iritani, T. Mitsukuchi, "Measurements of small ion mobility spectrum with multi-electrodes Gerdien condenser," Atmospheric Research Letters, vol. 2, no. 1, pp. 8-15, 1982.

[8] Y. S. Yue and G. Y. Chen, "Development of measurement apparatus of ion mobility and its changing law with different temperatures and humidities," High Voltage Engineering, vol. 41, no. 5, pp. 1696-1703, 2015.

[9] J. Bricard, M. Cabane, and G. Madelaine, "Study of the mobility of small ions in air by the flight time method," Planetary Electrodynamics, vol. 1, no. 2, pp. 243, 1969.

[10] R. G. Stearns, "Ion mobility measurements in a positive corona discharge," Journal of Applied Physics, vol. 67, no. 6, pp. 2789-2799, 1990.

[11] J. Bricard, M. Cabane, and G. Madelaine, "Formation of atmospheric ultrafine particles and ions from trace gases," Journal of Colloid and Interface Science, vol. 58, no. 1, pp. 113-124, 1977.

[12] J. Bricard, M. Cabane, G. Madelaine, and D. Vigla, "Formation and properties of neutral ultrafine particles and small ions conditioned by gaseous impurities of the air," Journal of Colloid and Interface Science, vol. 
39, no. 1, pp. 42-58, 1972.

[13] M. L. Huertas, A. M. Marty, J. Fontan, I. Alet, and G. Duffa, "Measurement of mobility and mass of atmospheric ions," Journal of Aerosol Science, vol. 2, no. 2, pp. 145-150, 1971.

[14] J. T. Ouyang, Z. L. Zhang, and Z. L. Peng, "Electromagnetic radiation from negative corona discharge in air," High Voltage Engineering, vol. 38, no. 9, pp. 2237-2241, 2012.

[15] Y. P. Liu, S. L. Huang, and L. Zhu, "Influence of humidity and air pressure on the ion mobility based on drift tube method," CSEE Journal of Power \& Energy Systems, vol. 1, no. 3, pp. 37-41, 2015.

[16] X. Liu, "The negative corona discharge electron source for ion mobility spectrometry," Ph.D. dissertation, Dept. Envir. Eng., HUST. Univ., Wuhan, China, 2012.

[17] K. Nagato and O. Toshio, "Atmospheric ion mobility spectra near the ground," Planetary \& Space Science, vol. 36, no. 2, pp. 163-176, 1988.

[18] Y. M. Ji, B. Zhang, and J. L. He, "Structure analysis and result correction of ion mobility measurement apparatus in air," High Voltage Engineering, vol. 40, no. 6, pp. 1768-1774, 2014.

[19] S. Liu, C. Q. Huang, C. Y. Shen, "Asymmetric Control Method for Ion Shutter and the Resolution Improvement of Ion Mobility Spectrum," Spectroscopy and Spectral Analysis, vol. 33, no. 11, pp. 28812885, 2013.

[20] T. Aschwanden, Swarm Parameters in SF6 and SF6/N2 Mixtures Determined from a Time-Resolved Discharge Study. New York: Pergamon Press, 1984.

[21] A. G. Arson, and I. M. Bortnik, "Mobility of ions in SF6," in Proceedings of 6th International Conference on Gas Discharges, 1980, pp. 165-167.

[22] R. T. Waters, O. Farish, and O. Ibrahim, "positive and negative mean ion mobilities in corona discharges in SF6 and mixtures," in Proceedings of 7th International Conference on Gas Discharges and Their Application, 1982, pp. 251-254.

[23] L. N. Tuong, D. Masayuki, S. R. Wang, "The effect of oxygen vacancy on the oxide ion mobility in $\mathrm{LaAlO}_{3}$-based oxides," Solid State Ionics, vol. 130, no. 1, pp. 229-241, 2000.

[24] A. V. Kosarim, B. M. Smirnov, M. Capitelli, "Determination of concentration of excited oxygen atoms on the basis of ion mobility in atmospheric plasma," International Journal of Mass Spectrometry, vol. 253, no. 1, pp. 22-29, 2006.

[25] C. A. Hill, C. L. P. Thomas, "A pulsed corona discharge switchable high resolution ion mobility spectrometer-mass spectrometer," Analyst, vol. 128, no. 1, pp. 55-60, 2003.

[26] A. Ebrahimi, M. T. Jafari, "Negative corona discharge-ion mobility spectrometry as a detection system for low density extraction solvent-based dispersive liquid-liquid microextraction," Talanta, vol. 134, pp. 724-731, 2015.

[27] R. Cumeras, et al, "Review on ion mobility spectrometry. Part 1: current instrumentation," Analyst. vol. 140, no. 5, pp. 1376-1390, 2015.

[28] M. Sabo, S. Matejc ík, "Ion mobility spectrometry for monitoring high-purity oxygen," Analytical chemistry, vol. 83, no. 6, pp. 1985-1989, 2011.

[29] M. Sabo, J. Páleník, M. Kučera, "Atmospheric pressure corona discharge ionisation and ion mobility spectrometry / mass spectrometry study of the negative corona discharge in high purity oxygen and oxygen/nitrogen mixtures," International Journal of Mass Spectrometry, vol. 293, no. 1, pp. 23-27.19851989, 2010.

[30] J. De Urquijo, "Measurement of negative ion mobilities in oxygen at high pressures with a pulsed Townsend technique," Revista Mexicana de Física, vol. 33, no. 1, pp. 5-12, 1988.

[31] G. A. Eiceman, Z. Karpas, and H. H. Hill Jr, Ion Mobility Spectrometry. Florida: CRC Press, 2013.

[32] G. A. Eiceman, E. G. Nazarov, J. E. Rodriguez, and J. A. Stone, "Analysis of a drift tube at ambient pressure: Models and precise measurements in ion mobility spectrometry," Review of Scientific Instruments, vol. 72, no. 9, pp. 3610-3621, 2001.

[33] P. S. Maruvada, Corona Performance of HighVoltage Transmission Lines, London, UK: Research Studies Press Ltd, 2000, pp. 84.

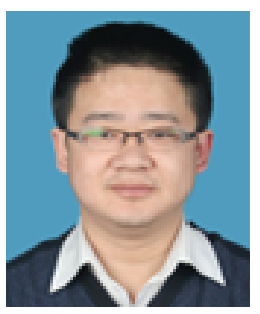

Yun-Peng Liu(M' 15) He was born in Jinzhai, Anhui Province, China, on April 1, 1976. He received his B.Sc. degree in electrical engineering from the North China Electric Power University, Baoding, China, in 1999 and $\mathrm{Ph} . \mathrm{D}$. from the same university in 2005. Currently, he is a doctoral supervisor and a professor in North China Electric Power University, Baoding, China. His research interests focus on UHV transmission and fault detection and diagnosis of electrical power equipment.

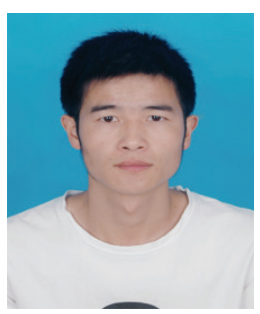

Shi-long Huang He received the B.Sc. degree from the North China Electric Power University, Baoding, China, in 2014. Now, he is pursuing the Ph.D. degree in the school of Electrical Engineering from North China Electric Power University. His research interests focus on UHV power transmission. 